\title{
Impact of tumor volume doubling time on post- metastatic survival in bone or soft-tissue sarcoma patients treated with metastasectomy and/or radiofrequency ablation of the lung
}

\author{
This article was published in the following Dove Press journal: \\ OncoTargets and Therapy \\ 31 January 2017 \\ Number of times this article has been viewed
}

\author{
Tomoki Nakamura' \\ Akihiko Matsumine' \\ Motoshi Takao² \\ Atsuhiro Nakatsuka ${ }^{3}$ \\ Takao Matsubara' \\ Kunihiro Asanuma' \\ Akihiro Sudo' \\ 'Department of Orthopedic \\ Surgery, Mie University Graduate \\ School of Medicine, ${ }^{2}$ Department \\ of Thoracic Surgery, ${ }^{3}$ Department \\ of Interventional Radiology, Mie \\ University Hospital, Tsu, Japan
}

\begin{abstract}
Metastasectomy represents the standard treatment for improving survival in patients with lung metastases (LMs) from bone (BS) or soft-tissue sarcoma (STS). Recently, radiofrequency ablation (RFA) of the LMs has been proved to be a useful option which can promise the similar effect to metastasectomy. The aim of this study was to determine prognostic factors, including tumor volume doubling time (TVDT), for post-metastatic survival in BS and STS patients treated with metastasectomy and/or RFA of the lung. Forty-eight patients with LMs were retrospectively reviewed. The mean age of the patients at the time of LMs was 56 years. The cohort comprised 27 male and 21 female patients. Eight of the 48 patients had LMs at the point of initial presentation. The mean follow-up period after commencing the treatment for LMs was 37 months. The mean maximum diameter of the initial LMs was $11 \mathrm{~mm}$. The mean number of LMs was 4. The TVDT was calculated using a method originally described by Schwartz. At last follow-up, 5 patients had no evidence of disease, 3 patients were still alive with disease, and 32 patients had died of disease. The 3-year and 5-year post-metastatic survival rates were $32 \%$ and $16.8 \%$, respectively. In a Cox univariate analysis, the size $(P=0.04)$ and number of LMs $(P<0.001)$, disease-free interval $(P=0.04)$, curability of the initial LMs $(P<0.001)$, and TVDT $(P<0.001)$ were significantly identified as factors which affect prognosis. In the multivariate analysis, TVDT $(P<0.001)$ and curability of the initial LMs $(P<0.001)$ were confirmed as independent predictors of survival. There was a significant association between the number and curability of the initial LMs $(P<0.001)$. In conclusion, metastasectomy and/or RFA of LMs is recommended for improving survival. However, TVDT and the curability of the LMs should be taken into consideration.
\end{abstract}

Keywords: sarcoma, lung metastasis, curability post-metastatic survival, tumor volume doubling time

\section{Introduction}

Several relevant prognostic factors (PFs) have been defined for bone (BS) or softtissue sarcoma (STS) after lung metastasis (LM). ${ }^{1-12}$ Metastasectomy represents the standard treatment for improving survival in patients with LMs from BS or STS. ${ }^{1-7}$ Recently, radiofrequency ablation (RFA) of the lung has proved to be a useful option which promise a similar outcome to metastasectomy. ${ }^{13-16}$ Age, histological tumor grade, disease-free interval (DFI), number of LMs, and tumor volume doubling time (TVDT) are also predictive factors of survival. ${ }^{1-12}$ The TVDT is an accurate and reproducible method for quantifying the rate and pattern of tumor growth in individual patients. ${ }^{8-12}$
Correspondence: Tomoki Nakamura Department of Orthopedic Surgery, Mie University Graduate School of Medicine, 2-I74 Edobashi, Tsu 5I 48507, Mie, Japan Tel +8I 5923 3I 5022

Fax $+8 \mid 5923$ 3 52II

Email tomoki66@clin.medic.mie-u.ac.jp 
We previously reported that a shorter TVDT, evaluated using chest computed tomography (CT) scanning, was associated with reduced post-metastatic survival in sarcoma patients. ${ }^{8}$ However, we included patients who were not treated with metastasectomy and/or RFA of the lung, in addition to those patients who underwent these treatments.

The aim of this study was to determine PFs, including TVDT, for post-metastatic survival in BS or STS patients treated with metastasectomy and/or RFA for the LMs.

\section{Materials and methods Inclusive patients}

Fifty-six patients with LMs from BS or STS were treated with metastasectomy and/or RFA of the lung between 2002 and 2015 . Seven patients $(12.5 \%)$ were excluded from the study because CT scan was performed only 1 time before treatment for LMs, although scanning at 2 points of time is necessary for measuring the TVDT. Another patient (1.8\%) with Ewing sarcoma, who had LMs at the time of initial screening, was also excluded from the study because all of the LMs disappeared after systemic neo- and adjuvant chemotherapy without metastasectomy or RFA (the patient underwent metastasectomy after 5 years due to relapsed LMs). In total, 48 patients (85.7\%) were retrospectively reviewed. Although 27 (56.2\%) of 48 patients were reviewed in a previous study of TVDT, the effect of surgical and interventional procedure was not taken into consideration for analysis of survival. ${ }^{8}$ This study was approved by the institutional review board of Mie University Hospital. Written informed consent was obtained from all patients for this study including their data to be used in the study.

\section{Method of measuring TVDT}

All 48 patients had measurable LMs in at least 2 sequential chest CT images taken $\geq 14$ days apart, before commencing treatment. No patient received chemotherapy during the period of measurement. The TVDT was calculated using a method originally described by Schwartz. ${ }^{9}$ The tumor volume was calculated as follows, assuming the tumor to have a spheroidal shape:

$$
V=4 / 3 \times \pi \times(a / 2)^{3}
$$

where $a$ indicates the maximum tumor diameter. The TVDT was calculated using the following equation:

$$
\operatorname{TVDT}=\left(T_{2}-T_{1}\right) \times \log 2 / \log V_{2}-\log V_{1}
$$

where $T_{2}-T_{1}$ represents the time interval between 2 measurements and $V_{1}$ and $V_{2}$ denote the TVDT at 2 points of measurement. Treatment plans for the LMs were determined by the members of a multidisciplinary team.

\section{Indication for metastasectomy and RFA}

Thoracic surgeons assessed the resectability of the LMs after considering the number and location of LMs and the patient's general condition. The indication for metastasectomy was as follows: (a) no extrapulmonary metastases, (b) well-controlled primary tumor, and (c) possible complete resection of all metastases.

Interventional radiologists assessed the indication for RFA. The indication for RFA was as follows: (a) patients with multiple LMs who were predicted to have respiratory loss after metastasectomy, (b) tumors which were not close to vessels or bronchus, and (c) patients refusing metastasectomy, although metastasectomy was recommended for the initial LMs after the confirmation of diagnosis, unless there were obvious multiple LMs.

We defined curability as complete or incomplete treatment of all initial LMs on CT with metastasectomy or RFA. Complete treatment meant all initial LMs on CT were treated with metastasectomy/RFA. Incomplete treatment meant all initial LMs on CT were not treated with metastasectomy or RFA.

The main aim of this study was to use univariate and multivariate analyses to examine PFs associated with survival in BS or STS patients with LMs.

\section{Statistical analyses}

All statistical analyses were conducted using the StatView program for Windows, software version 5.0 (SAS Institute Inc., Cary, NC, USA). Mann-Whitney $U$ tests (quantitative data) and chi-square or Fisher's exact tests (qualitative data) were performed to assess the statistical association between clinicopathological factors. The DFI was defined as the duration between the time the primary tumor was resected and the date LMs were initially detected (the DFI in patients with LMs at the point of initial presentation was defined as 0 month). Overall survival was defined as the duration between the time of the initial treatment for LMs and the date of death or last follow-up. Survival curves were estimated using the Kaplan-Meier method. Cumulative survival rates were compared using the log-rank test. Univariate and multivariate analyses were conducted using a Cox proportional hazards model to compare overall survival between patients. 
Probability $P$-values $<0.05$ were considered significant in all statistical analyses.

\section{Results}

\section{Patients clinicopathological characteristics}

In total, 48 patients with LMs from BS or STS were treated with metastasectomy and/or RFA. The mean age of the patients at the time of LMs was 56 years (range, 12-88 years). The cohort comprised 27 male $(56.3 \%)$ and 21 female (43.7\%) patients. Eight of the 48 patients (16.7\%) had LMs at the point of initial presentation. The mean follow-up period after commencing the treatment for LMs was 37 months (range, 6-158 months). The mean maximum diameter of the initial LMs was $11 \mathrm{~mm}$ (range, 3-35 mm). The mean number of LMs was 4 (range, 1-17). The distribution of LMs, according to histological subtype, was as follows: leiomyosarcoma, $n=11$; undifferentiated pleomorphic sarcoma/ malignant fibrous histiocytoma, $n=7$; osteosarcoma, $n=5$; synovial sarcoma, $n=5$; myxofibrosarcoma, $n=4$; extraskeletal chondrosarcoma, $n=3$; malignant peripheral nerve sheath tumor, $n=3$; chondrosarcoma, $n=2$; myxoid liposarcoma, $n=2$; malignant granular cell tumor, $n=2$; and other tumor, $n=4$. The median and mean DFI for all patients, including those patients with LMs at the point of initial presentation $(n=8)$, was 10.8 months and 22.3 months, respectively. The median and mean DFI for the 40 patients $(83.3 \%$ ) who developed LMs after resection of the primary tumor was 14.3 months and 26.7 months, respectively. The median and mean TVDT for all 48 patients was 35 days and 69 days, respectively (range, 10-506 days). Twenty-seven (56.3\%) and 38 (79.2\%) of the 48 patients underwent metastasectomy and RFA of the lung for LMs, respectively (metastasectomy alone, $n=10$; RFA of the lung alone, $n=21$; both metastasectomy and RFA of the lung, $n=17)$. Twenty-five patients $(52.1 \%)$ received chemotherapy for LMs (Table 1).

\section{Patient survival and risk factors associated with survival}

At last follow-up, 15 patients (31.3\%) had no evidence of disease, 3 patients $(6.2 \%)$ were still alive with disease, and 30 patients $(62.5 \%)$ had died of disease. Causes of death included LMs $(n=25)$, local tumor progression $(n=1)$, liver metastasis $(n=1)$, brain metastasis $(n=1)$, adrenal metastasis $(n=1)$, and intraabdominal metastasis $(n=1)$. The 3-year and 5-year post-metastatic survival rates were $32 \%$ and $16.8 \%$, respectively (Figure 1). In a Cox univariate analysis, the size $(P=0.04)$ and number of LMs $(P<0.001)$, DFI
Table I Patients background

\begin{tabular}{ll}
\hline Characteristic & Total \\
\hline Age & 56 \\
Mean (years) & $12-88$ \\
$\quad$ Range (years) & \\
Sex & 27 \\
$\quad$ Male & 21 \\
$\quad$ Female & \\
Maximum diameter of metastases & 11 \\
$\quad$ Mean (mm) & $3-35$ \\
Range (mm) & \\
Number of metastases & 4 \\
$\quad$ Mean & $1-17$ \\
$\quad$ Range & \\
Tumor volume doubling time & 69 \\
Mean (days) & 35 \\
Median (days) & $10-506$ \\
Range (days) & \\
Treatment for lung metastases & 10 \\
Metastasectomy alone & 21 \\
RFA alone & 17 \\
Both & \\
Chemotherapy for lung metastases & 25 \\
Yes & 23 \\
No &
\end{tabular}

Abbreviation: RFA, radiofrequency ablation.

( $P=0.04)$, curability of the initial LMs $(P<0.001)$, and TVDT $(P<0.001)$ were identified as significant PFs (Table 2). In the multivariate analysis, TVDT $(P<0.001)$ and curability of the initial LMs $(P<0.001)$ were confirmed as independent predictors of survival (Table 3 ). There was a significant association between the number and curability of the initial LMs $(P<0.001)$. Conversely, age, gender, the size of the LMs, DFI, and TVDT did not correlate with curability (Table 4).

We subsequently analyzed the association between TVDT, curability, and survival. Patients with incomplete

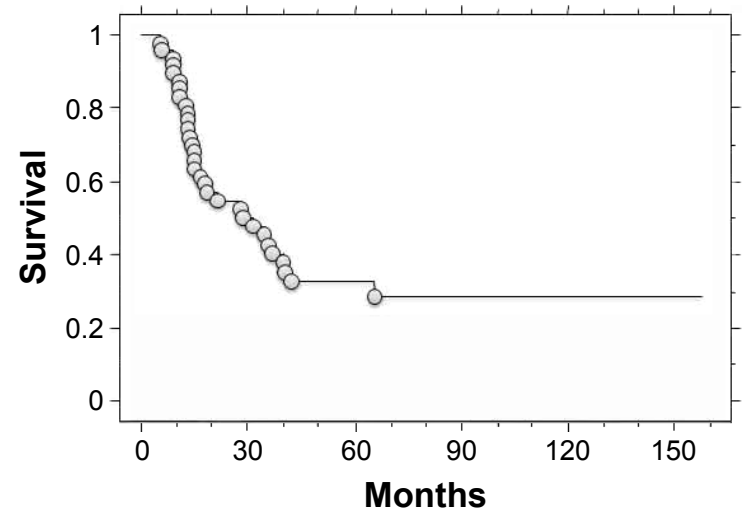

Figure I Kaplan-Meier curve of post-metastatic survival in bone or soft-tissue sarcoma patients $(n=48)$ treated with metastasectomy and/or radiofrequency ablation of the lung. 
Table 2 Univariate analysis for survival

\begin{tabular}{llll}
\hline Variables & HR & $\mathbf{9 5 \% ~ C l}$ & P-value \\
\hline $\begin{array}{l}\text { Age (years) } \\
\text { Gender }\end{array}$ & 1.011 & $0.991-1.031$ & 0.28 \\
$\quad \begin{array}{lll}\text { Female } \\
\text { Metastasis at }\end{array}$ & 0.899 & $0.440-1.839$ & 0.77 \\
$\quad$ No & 1.079 & $0.412-2.822$ & 0.88 \\
Size (mm) & 1.044 & $1.002-1.088$ & 0.04 \\
Number & 1.125 & $1.031-1.227$ & 0.008 \\
Curability & & & \\
$\quad$ Complete & 0.291 & $0.142-0.597$ & 0.0008 \\
DFI (months) & 0.98 & $0.962-0.999$ & 0.04 \\
TVDT (days) & 0.966 & $0.948-0.985$ & 0.0004 \\
\hline
\end{tabular}

Abbreviations: $\mathrm{HR}$, hazard risk; $\mathrm{Cl}$, confidence interval; $\mathrm{DFI}$, disease-free interval; TVDT, tumor volume doubling time.

treatment and a shorter TVDT had a poorer disease-specific survival ( $0.0 \%$ at 2 years) compared to patients with complete treatment and a longer TVDT $(93.3 \%$ at 2 years and $85.6 \%$ at 3 years; $P<0.001)$. Patients with incomplete treatment and a shorter TVDT also had a poorer disease-specific survival compared to patients with complete treatment and a shorter TVDT $(42.9 \%$ at 2 years and $28.6 \%$ at 3 years; $P<0.01)$ or patients with incomplete treatment and a longer TVDT ( $51.9 \%$ at 2 years and $41.6 \%$ at 3 years; $P=0.02$ ). There was no significant prognostic difference between patients with incomplete treatment and a longer TVDT and patients with complete treatment and a shorter TVDT. Patients with complete treatment and a longer TVDT had a significantly better survival rate compared to patients with incomplete treatment and a longer TVDT $(P<0.001)$ or patients with complete treatment and a shorter TVDT $(P<0.001$; Figure 2$)$.

\section{Discussion}

Of all the patients diagnosed with BS or STS, between $10 \%$ and $38 \%$ of patients presented with clinically detectable metastases. ${ }^{7,17,18}$ Regarding the location of these metastases, between $62 \%$ and $83 \%$ of patients had LMs. ${ }^{17,19,20}$ Lung metastasectomy is reportedly a pivotal therapeutic option, providing patients with long survival times. ${ }^{3-7}$ Recently,

Table 3 Multivariate analysis for survival

\begin{tabular}{llll}
\hline Variables & HR & $\mathbf{9 5 \% ~ C l}$ & P-value \\
\hline Size $(\mathrm{mm})$ & 1.037 & $0.98-1.097$ & 0.21 \\
Number & 0.997 & $0.869-1.144$ & 0.97 \\
Curability & & & \\
$\quad$ Complete & 0.165 & $0.058-0.466$ & 0.0007 \\
DFI (months) & 1.003 & $0.987-1.020$ & 0.68 \\
TVDT (days) & 0.949 & $0.925-0.972$ & $<0.000$ I \\
\hline
\end{tabular}

Abbreviations: $\mathrm{HR}$, hazard risk; $\mathrm{Cl}$, confidence interval; $\mathrm{DFl}$, disease-free interval; TVDT, tumor volume doubling time.
Table 4 Predictive factors which related with curability

\begin{tabular}{|c|c|c|c|}
\hline \multirow[t]{2}{*}{ Variables } & \multicolumn{2}{|c|}{ Resectability } & \multirow[t]{2}{*}{$P$-value } \\
\hline & $\begin{array}{l}\text { Complete } \\
(n=30)\end{array}$ & $\begin{array}{l}\text { Incomplete } \\
(n=18)\end{array}$ & \\
\hline \multicolumn{4}{|l|}{ Age (years) } \\
\hline Mean & 52 & 63 & 0.08 \\
\hline \multicolumn{4}{|l|}{ Gender } \\
\hline Male & 16 & 11 & 0.6 \\
\hline Female & 14 & 7 & \\
\hline \multicolumn{4}{|c|}{ Metastasis at presentation } \\
\hline No & 26 & 14 & 0.42 \\
\hline Yes & 4 & 4 & \\
\hline \multicolumn{4}{|l|}{ Size $(\mathrm{mm})$} \\
\hline Mean & 10.3 & 12.1 & 0.28 \\
\hline Number & 2.2 & 6.3 & $<0.000$ \\
\hline DFI (months) & 26.8 & 14.5 & 0.12 \\
\hline \multicolumn{4}{|l|}{ TVDT (days) } \\
\hline Mean & 85.1 & 42.2 & 0.62 \\
\hline
\end{tabular}

Abbreviations: DFI, disease-free interval; TVDT, tumor volume doubling time.

RFA of the lung has been accepted as a relatively safe and useful therapeutic option for the treatment of unresectable lung cancer and metastatic tumors. ${ }^{13-16}$ However, even after an apparent complete resection or ablation of the metastases, in $40 \%-80 \%$ of patients, the metastases had recurred. ${ }^{3,20}$ In our case series, the 3-year and 5-year post-metastatic survival rates were $32 \%$ and $16.8 \%$, respectively. The present findings are consistent with those of previously published reports. ${ }^{1-7}$

From the multivariate analysis, we identified TVDT and curability of the initial LMs as independent predictors of survival. There was a significant association between the number and curability of the initial LMs. Interestingly, patients with incomplete treatment and a shorter TVDT had a poorer disease-specific survival compared to patients with

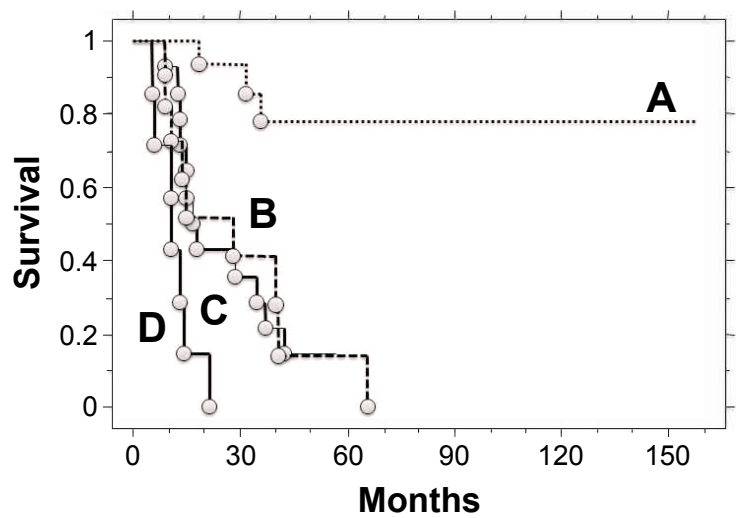

Figure 2 Kaplan-Meier curves of post-metastatic survival in bone or soft-tissue sarcoma patients $(n=48)$ with (A) complete treatment and a tumor volume doubling time (TVDT) of $>30$ days, (B) incomplete treatment and a TVDT of $>30$ days, (C) complete treatment and a TVDT of $\leq 30$ days, and (D) incomplete treatment and a TVDT of $\leq 30$ days. 
incomplete treatment and a longer TVDT or patients with complete treatment and a shorter TVDT. Furthermore, there was no significant prognostic difference between patients with incomplete treatment and a longer TVDT and patients with complete treatment and a shorter TVDT. Therefore, TVDT and curability of the LMs should be taken into consideration when planning metastasectomy and/or RFA of the lung. Our findings suggest that those patients with LMs who have a longer TVDT should be considered for metastasectomy and/or RFA of the lung even if the lesions are multiple and/or bilateral. Furthermore, if all detectable metastases are controllable, then metastasectomy and/or RFA of the lung should be considered even if the TVDT is short.

Small pulmonary nodules $(<5 \mathrm{~mm})$ are easily detectable in STS patients because of highly improved CT technologies. ${ }^{21}$ In the present study, BS or STS patients were classified into 2 groups according to a TVDT threshold of 30 days. This strategy was selected because the TVDT is simple to calculate, a TVDT of 30 days has important indications for understanding tumor aggressiveness, and the median TVDT was 35 days in our case series. Based on the Schwartz equation, LMs of $3 \mathrm{~mm}$ or $5 \mathrm{~mm}$ in patients with a TVDT of 30 days would grow to approximately $6 \mathrm{~mm}$ or $10 \mathrm{~mm}$ in 90 days. ${ }^{9}$ Similarly, LMs of $3 \mathrm{~mm}$ or $5 \mathrm{~mm}$ in patients with a TVDT of 10 days would grow to approximately $6 \mathrm{~mm}$ or $10 \mathrm{~mm}$ in 30 days. Therefore, subsequent CT scans in patients with pulmonary nodules should be taken within 3 months. Metastasectomy and/or RFA of the lung is recommended if the nodules have increased in size at follow-up CT. In that time, TVDT and the curability of the LMs should be taken into consideration. Our results also suggest that patients with poor curability and a shorter TVDT have a significantly higher risk of mortality despite metastasectomy and/or RFA. Therefore, systemic chemotherapy should be considered in those patients. We recommend a TVDT of 30 days as 1 indication of a clinical decision.

There are some limitations to this study: (1) the study population was small for considering associations between different histological tumor types and clinical outcomes, (2) the study had a retrospective design, and (3) treatment was administered at the discretion of the physician, although indications of metastasectomy and/or RFA of the lung were determined after the discussion with the members of a multidisciplinary team. For example, metastasectomy and/or RFA of the lung may be limited in some patients who had multiple metastases by physician's decision without calculation of TVDT. Further prospective studies will be necessary to validate our findings.

\section{Conclusion}

Metastasectomy and/or RFA of the lung is recommended for LMs if the nodules have increased in size. For this procedure, TVDT and the curability of the LMs should be taken into consideration.

\section{Disclosure}

The authors report no conflicts of interest in this work.

\section{References}

1. Casson AG, Putnam JB, Natarajan G, et al. Five-year survival after pulmonary metastasectomy for adult soft tissue sarcoma. Cancer. 1992;69(3):662-668.

2. Billingsley KG, Burt ME, Jara E, et al. Pulmonary metastases from soft tissue sarcoma: analysis of patterns of disease and postmetastasis survival. Ann Surg. 1999;229(5):602-610.

3. Weiser MR, Downey RJ, Leung DH, Brennan MF. Repeat resection of pulmonary metastases in patients with soft-tissue sarcoma. J Am Coll Surg. 2000;191(2):184-190.

4. van Geel AN, Hoekstra HJ, van Coevorden F, Meyer S, Bruggink ED, Blankensteijn JD. Repeated resection of recurrent pulmonary metastatic soft tissue sarcoma. Eur J Surg Oncol. 1994;20(4):436-440.

5. Billingsley KG, Lewis JJ, Leung DH, Casper ES, Woodruff JM, Brennan MF. Multifactorial analysis of the survival of patients with distant metastasis arising from primary extremity sarcoma. Cancer. 1999; 85(2):389-395.

6. Putnam JB Jr, Roth JA, Wesley MN, Johnston MR, Rosenberg SA Analysis of prognostic factors in patients undergoing resection of pulmonary metastases from soft tissue sarcomas. J Thorac Cardiovasc Surg. 1984;87(2):260-268.

7. Harting MT, Blakely ML, Jaffe N, et al. Long-term survival after aggressive resection of pulmonary metastases among children and adolescents with osteosarcoma. J Pediatr Surg. 2006;41(1):194-199.

8. Nakamura T, Matsumine A, Matsubara T, Asanuma K, Uchida A, Sudo A Clinical impact of the tumor volume doubling time on sarcoma patients with lung metastases. Clin Exp Metastasis. 2011;28(8):819-825.

9. Schwartz M. A biomathematical approach to clinical tumor growth Cancer. 1961;14:1272-1294.

10. Roth JA, Putnam JB Jr, Wesley MN, Rosenberg SA. Differing determinations of prognosis following resection of pulmonary metastases from osteogenic and soft tissue sarcoma patients. Cancer. 1985;55(6): 1361-1366.

11. Joseph WL, Morton DL, Adkins PC. Prognostic significance of tumor doubling time in evaluating operability in pulmonary metastatic disease. J Thorac Cardiovasc Surg. 1971;61(1):23-32.

12. Blomqvist C, Wiklund T, Tarkkanen M, Elomaa I, Virolainen M. Measurement of growth rate of lung metastases in 21 patients with bone or soft-tissue sarcoma. Br J Cancer. 1993;68(2):414-417.

13. Saumet L, Deschamps F, Marec-Berard P, et al. Radiofrequency ablation of metastases from osteosarcoma in patients under 25 years: the SCFE experience. Pediatr Hematol Oncol. 2015;32(1):41-49.

14. Koelblinger C, Strauss S, Gillams A. Outcome after radiofrequency ablation of sarcoma lung metastases. Cardiovasc Intervent Radiol. 2014;37(1):147-153.

15. Palussière J, Italiano A, Descat E, et al. Sarcoma lung metastases treated with percutaneous radiofrequency ablation: results from 29 patients. Ann Surg Oncol. 2011;18(13):3771-3777.

16. Nakamura T, Matsumine A, Yamakado K, et al. Lung radiofrequency ablation in patients with pulmonary metastases from musculoskeletal sarcomas. Cancer. 2009;115(16):3774-3781.

17. Kane JM, Finley JW, Driscoll D, Kraybill WG, Gibbs JF. The treatment and outcome of patients with soft tissue sarcomas and synchronous metastases. Sarcoma. 2002;6(2):69-73. 
18. Pollock RE, Karnell LH, Menck HR, Winchester DP. The national cancer data base report on soft tissue sarcoma. Cancer. 1996;78(10): 2247-2257.

19. Vezeridis MP, Moore R, Karakousis CP. Metastatic patterns in soft tissue sarcomas. Arch Surg. 1983;118(8):915-918.
20. Nakamura T, Matsumine A, Matsubara T, et al. Retrospective analysis of metastatic sarcoma patients. Oncol Lett. 2011;2(2):315-318.

21. Nakamura T, Matsumine A, Niimi R, et al. Management of small pulmonary nodules in patients with sarcoma. Clin Exp Metastasis. 2009; 26(7):713-718.

\section{Publish your work in this journal}

OncoTargets and Therapy is an international, peer-reviewed, open access journal focusing on the pathological basis of all cancers, potential targets for therapy and treatment protocols employed to improve the management of cancer patients. The journal also focuses on the impact of management programs and new therapeutic agents and protocols on

\section{Dovepress}

patient perspectives such as quality of life, adherence and satisfaction. The manuscript management system is completely online and includes a very quick and fair peer-review system, which is all easy to use. Visit http://www.dovepress.com/testimonials.php to read real quotes from published authors.

Submit your manuscript here: http://www.dovepress.com/oncotargets-and-therapy-journa 